\title{
Protocol
}

\section{Exploration of sedentary behaviour among general practitioners: protocol for a mixed methods study}

\author{
Richard S. Mayne ${ }^{1 *}$, Nigel D. Hart ${ }^{2}$, Neil Heron ${ }^{3}$
}

\begin{abstract}
${ }^{1}$ Faculty of Life and Health Sciences, ${ }^{2}$ Centre for Medical Education, ${ }^{3}$ Centre for Public Health, Queen's University Belfast, Belfast, United Kingdom
\end{abstract}

Received: 23 October 2020

Accepted: 06 January 2020

\section{*Correspondence:}

Dr. Richard S. Mayne,

E-mail: rmayne02@qub.ac.uk

Copyright: @ the author(s), publisher and licensee Medip Academy. This is an open-access article distributed under the terms of the Creative Commons Attribution Non-Commercial License, which permits unrestricted non-commercial use, distribution, and reproduction in any medium, provided the original work is properly cited.

\section{ABSTRACT}

Background: Many general practitioners (GPs) are sedentary for most of their working day. Levels of sedentary behaviour may have been exacerbated by increased use of telemedicine in light of the COVID-19 pandemic, as this is traditionally performed while sitting down. Excessive sedentary behaviour is associated with many adverse health outcomes and increased all-cause mortality. This study will gain quantitative data on levels of sedentary behaviour among GPs and general practice specialty trainees (GPSTs), to identify to what extent general practice is a sedentary occupation, as well as qualitative data regarding the barriers and facilitators to reducing sedentary behaviour in the general practice setting.

Methods: The study follows a sequential, mixed-methods model. The first stage will involve the dissemination of a questionnaire survey, where participants self-estimate their sedentary behaviour on a working day and on a non-working day. The second stage will use thigh-worn accelerometers and a sleep/work log to obtain objective data regarding sedentary behaviour among a purposive subset of participants who responded to the questionnaire. The third stage will involve semi-structured interviews with a purposive subset of accelerometer study participants, analysed with the application of a theoretical framework regarding the acceptability of healthcare interventions.

Conclusions: This paper outlines a protocol for a sequential, mixed-methods study exploring sedentary behaviour among GPs and GPSTs. Findings of this study will shed light on the new ways of working as a result of the COVID19 pandemic, which will be relevant to clinicians working in similar primary care settings throughout the world.

Trial Registration: ClinicalTrials.gov Identifier: NCT04556695. Date of registration: $21^{\text {st }}$ September 2020.

Keywords: General practitioner, Primary care, Sedentary behaviour, Physical activity

\section{INTRODUCTION}

Sedentary behaviour is defined as when someone is awake, in a sitting, lying or reclining posture, with low energy expenditure. ${ }^{1}$ It is acknowledged that sedentary behaviour is associated with multiple adverse health outcomes, including mental health issues, obesity, type 2 diabetes, multiple forms of cardiovascular disease and dementia, as well as breast, colorectal, endometrial and ovarian cancer. $^{2-4}$ As a result of these adverse health outcomes, sedentary behaviour is associated with increased all-cause mortality, even when allowing for confounding variables. ${ }^{4,5}$ It is important to note that non-exercise activity thermogenesis (NEAT), i.e. movement and posture changes during activities of daily living, overall accounts for greater energy expenditure than deliberate exercise in the vast majority of the population. ${ }^{6}$ Although it is debatable the extent to which levels of sedentary behaviour can be attenuated by physical activity, sedentary behaviour is an independent risk factor for increased mortality. $5,7,8$

Sedentary behaviour and physical inactivity have significant economic costs. Conservatively estimated, 
physical inactivity was calculated to cost the United Kingdom (UK) National Health Service (NHS) $£ 1.4$ billion, with worldwide costs estimated at $£ 41.9$ billion in 2013. ${ }^{9,10}$ More specifically, sedentary behaviour was estimated to cost the UK NHS $£ 0.7$ billion in 2016-2017. ${ }^{11}$ A total of 69,276 deaths could potentially have been avoided in the UK if sedentary behaviour was eliminated. ${ }^{11}$ In light of these findings, 2019 UK physical activity guidelines state that through all stages of life, individuals should minimise their sedentary behaviour, and break up periods of sedentary behaviour where possible. ${ }^{12}$

General practitioners (GPs) have high levels of patient contact and constitute a significant portion of the medical workforce in most developed countries. ${ }^{13}$ Primary care has been described as "the cornerstone" of the National Health Service in the United Kingdom, providing over 300 million patient consultations per year. ${ }^{14}$ This allows GPs to play an important role in primary and secondary prevention, by providing evidence-based lifestyle guidance to patients. Despite this, physical activity promotion and guidance from GPs to their patients has generally been shown to be poor. ${ }^{15,16}$ Numerous studies have demonstrated that GPs who are more physically active are more likely to recommend physical activity to their patients. ${ }^{17-21}$ Patients are also more likely to make healthy lifestyle changes recommended by their doctor if they believe their doctor follows the health advice themselves. ${ }^{22-24}$ It could therefore be argued that reducing sedentary behaviour and increasing physical activity among GPs could lead to health benefits for both GPs, at an individual level, and their patients, at a population level.

Given that many GPs spend the majority of their waking lives in work, it is important to consider their levels of sedentary behaviour and physical activity in the workplace. Changes caused by the COVID-19 pandemic have resulted in a reduction in face-to-face consulting by GPs, with a corresponding increase in remote consulting by telephone and video, tasks traditionally performed while sitting down. ${ }^{25}$

It is only in recent years that sedentary behaviour has been identified and defined as a separate entity from physical inactivity. ${ }^{1}$ For this reason, there have been few previous studies examining sedentary behaviour among GPs. ${ }^{26,27} \mathrm{~A}$ limitation of previous studies is that they primarily focused on self-reported physical activity of GPs, with just one question addressing levels of sedentary behaviour. ${ }^{26,27}$ They also took place prior to the COVID-19 pandemic, which has resulted in significant changes to the working patterns and practices of many GPs.

There have been numerous studies examining levels of physical activity among GPs. Although there is variation in the personal health practices of GPs between different countries and regions throughout the world, the majority of GPs do not meet current physical activity guidelines. ${ }^{26,28,29}$ Some studies of physical activity and sedentary behaviour among doctors have differentiated between different medical specialties, work environments and levels of training. When focusing specifically on GP trainees, those who were working in a hospital setting (in different medical specialties to general practice), had greater levels of physical activity and less sedentary behaviour than trainees in general practice posts. ${ }^{26}$ Levels of physical activity have also been shown to change during different stages of medical training, with a reduction in physical activity as individuals move from studying at medical school to working as a doctor. ${ }^{29}$

This study aims to gain quantitative data on levels of sedentary behaviour and physical activity among GPs and general practice specialty trainees (GPSTs), to identify to what extent general practice is a sedentary occupation. This study also aims to gather qualitative data regarding the barriers and facilitators to reducing sedentary behaviour in the general practice setting.

To achieve these aims the research questions are as follows: what are the current levels of sedentary behaviour among GPs and GPSTs who work in either general practice or hospital settings on a typical working day?, what are the current levels of sedentary behaviour among GPs and GPSTs who work in either general practice or hospital settings on a typical day off work?, are there differences in the levels of sedentary behaviour between GPs and GPSTs depending on their working environment, age and gender?, what proportion of GPs and GPSTs have access to an "active workstations" such as standing desks?, how have levels of sedentary behaviour been affected by changes due to the COVID-19 pandemic?, what are the current levels of knowledge among GPs and GPSTs regarding the health effects of excessive sedentary behaviour?, and what are the barriers and facilitators to reducing sedentary behaviour among GPs and GPSTs?

\section{METHODS}

\section{Study design overview}

The study follows a sequential, mixed-methods model based on an explanatory design. ${ }^{30}$ Research will be conducted in three stages.

\section{Stage 1: online questionnaire}

\section{Recruitment and data collection}

An initial online questionnaire, based on the international sedentary assessment tool (ISAT), will be distributed to general practitioners (GPs) and general practice specialty trainees (GPSTs) throughout Northern Ireland. ${ }^{31}$ GPs and GPSTs in Northern Ireland provide care for patients as part of the UK NHS, with working conditions similar to GPs and GPSTs throughout the rest of the UK. A link to the questionnaire will be disseminated by email via GP and GPST mailing lists, as well as by social media (Facebook and Twitter). Questionnaires have been used extensively to determine levels of sedentary behaviour among large 
population groups. Multi-item questionnaires with a relatively short recall period have been shown to be more reliable than single item questions and longer recall periods for the assessment of sedentary time. ${ }^{32}$ The ISAT questionnaire was formulated by including the most reliable and valid questions related to specific aspects of sedentary behaviour identified after a systematic review of 49 sedentary behaviour questionnaires. ${ }^{31}$ The questionnaire used for this study will obtain information regarding the age, gender and working practice of each participant, before asking specific questions regarding sedentary behaviour on a working day and on a nonworking day. Further questions will explore whether participants have access to an active workstation (such as a standing desk), whether their sedentary behaviour has changed as a result of the COVID-19 pandemic, and whether they would prefer more, the same, or less time sitting when in work. Similar previous surveys assessing physical activity and sedentary behaviour among GPs and GPSTs in the Republic of Ireland by Keohane et al and among GPs in Estonia by Suija et al obtained a response rate of 25-30\%, with 219 and 198 participants eligible for inclusion. ${ }^{26,27}$ As there are currently 1999 GPs and GPSTs currently working in Northern Ireland, a similar response rate for this survey would result in approximately 500 responses. Participants will be recruited voluntarily, with no obligation to take part. Recruitment will take place opportunistically online via email and social media, using GP mailing lists and social media pages. Prior to undertaking the online survey, information regarding the purposes and nature of the study will be provided to participants. Having read the necessary information, participants must provide their consent before completing the questionnaire. Participants will also be asked if they consent to being contacted for the purposes of further research, and will be asked to provide their contact details if they do so.

\section{Data analyses}

Survey responses giving quantitative data will be analysed to calculate the mean, standard deviation (SD) and median for continuous variables or number and percentage for categorical variables. Differences between GPs and GPSTs with low, moderate, and high levels of sedentary behaviour will be analysed with the Chi-square test. All tests will be 2-sided and statistical significance will be set at $\mathrm{p}<0.05$. Qualitative data will be summarised according to relevant descriptive themes.

\section{Stage 2: accelerometer study}

\section{Recruitment and data collection}

A purposive sample of approximately 20 questionnaire respondents will be asked to participate in the accelerometer study. This purposive sample will be based on responses to the online questionnaire. The aim will be to obtain a varied sample, based on questionnaire responses, by selecting individuals with a range of demographic characteristics and self-reported levels of sedentary behaviour. An Axivity AX3 accelerometer will be posted to each participant, along with adhesive waterproof dressings (Tegaderm roll, 3M, Saint-Paul, Minnesota, USA) and instructions on how to affix the accelerometer to the middle of the right thigh. Thigh-worn accelerometers are highly accurate for distinguishing between sedentary behaviour (such as sitting or lying down) and other behaviours (such as standing and physical activity). ${ }^{33}$ Axivity AX3 accelerometers have been validated for the estimation of sedentary time and show good equivalence with other brands of accelerometers. ${ }^{34}$ Consenting participants will be asked to wear the accelerometer using a waterproof, adhesive dressing on the lateral aspect of the middle of the right thigh (over the midpoint of the iliotibial band), continuously over a sevenday period. They will also be asked to complete a contemporaneous sleep/work log over the same time period. At the end of the seven days, the accelerometers and sleep/work log will be returned to the research team using a stamped, addressed envelope.

\section{Data analyses}

Accelerometers will be set up to capture triaxial acceleration data over the time period that they are worn at $50 \mathrm{~Hz}$ with a dynamic range of $\pm 8 \mathrm{~g}$. Specific details on accelerometer data processing and analysis can be found in a previous study which also used Axivity AX3 accelerometers. ${ }^{35}$ For inclusion in the final analysis, accelerometers will need to be worn for a minimum of four valid days. A valid day will require a minimum of 600 minutes of wear-time as required for previous accelerometer studies. ${ }^{36}$ Accelerometer data will be used to determine sedentary time and time spent during physical activity among eligible participants. Alongside the sleepwork logs, this will allow comparison of sedentary time and physical activity to be made between working days and days off work, as well as between participants.

\section{Stage 3: semi-structured interviews}

\section{Recruitment and data collection}

A purposive sample comprising participants of the accelerometer study will be asked to participate in semistructured interviews. This purposive sample will be based on the accelerometer data. The aim will be to obtain a varied sample, based on accelerometer data, by selecting individuals with a range of different levels of sedentary behaviour, demographic and workplace characteristics. The final number of participants will depend on the saturation of information. Participants will be provided with a participant information sheet, which will be sent via email. This will provide detail regarding the expected duration and format of the semi-structured interviews, as well as some of the types of questions that they are likely to be asked. A follow-up email will then ask potential participants if they are happy to participate in the semistructured interview, with arrangements made to work out 
a time that is convenient to the participant. Semi-structured interviews will take place once accelerometer data has been analysed and interpreted.

Semi-structured interviews will take place via telephone or webcam. Participants will be given feedback regarding their accelerometer data relating to their levels of physical activity and sedentary behaviour. Questions will examine participants' thoughts and opinions regarding sedentary behaviour and physical activity in the general practice setting. Further questions will explore participants' experiences of wearing accelerometers, their knowledge of and attitudes towards physical activity and sedentary behaviour and their thoughts or suggestions on interventions to reduce sedentary behaviour among GPs and GPSTs. Audio recordings and transcriptions of semistructured interviews will be stored securely using password protection and will only be available and accessible to the research team.

\section{Data analyses}

Audio recordings will be transcribed verbatim by members of the research team and analysed using qualitative methodologies. Initial coding will take place shortly after the first interviews have been conducted. This will allow emerging themes to be identified and explored in more depth in subsequent interviews. Recordings will be destroyed once transcription has taken place. Qualitative methods will be used to identify common themes from interview responses. It is important to consider the acceptability of healthcare interventions in determining whether people will engage with the intervention. For this reason a theoretical framework regarding the acceptability of healthcare interventions will be applied as described by Sekhon et al. ${ }^{37}$

\section{COVID-19 considerations}

In the original study design planned prior to the COVID19 pandemic, recruitment to the study was planned to take place in person, at regional study days for GPs and GPSTs throughout Northern Ireland. As a result of the COVID-19 pandemic, all face-to-face study days were cancelled indefinitely, with sessions taking place remotely instead. This required an amendment to the study design to allow for the entire study to take place remotely, in order to minimise potential for spread of SARS-CoV-2 infection. Accelerometers worn by the participants will not come into contact with patients or other individuals, as they will remain covered throughout the duration of the study. Accelerometers will be cleaned using appropriate cleaning solutions prior to being sent to and worn by study participants. Participation in the study will not interfere with day-to-day workload for the participants.

\section{Patient and public involvement}

During the conception of the study, discussions were held with GPs and GPSTs regarding the acceptability of each of the stages of the study for potential participants. Participants will be actively encouraged to engage constructively with the research team throughout each stage of the study. Participants who wear accelerometers will be given feedback regarding their own accelerometer data, as well as how it compared to the overall average.

\section{DISCUSSION}

There has been little previous research examining sedentary behaviour among GPs, despite established evidence of the harmful health effects of sedentary behaviour. $^{2,3}$ In the "new normal," during and post COVID-19, with the majority of patient interactions now taking place remotely, GPs may have less physical activity, and more sedentary time throughout the working day. ${ }^{25}$ This may lead to a consequential higher risk of the negative health outcomes associated with excessive sedentary behaviour. ${ }^{2-4}$ Reduced non-exercise activity thermogenesis, as a result of increased levels of sedentary behaviour, is one of the main reasons behind the steady increase in global average body mass index (BMI) levels over time. ${ }^{6,38}$ In 2016 the average global BMI was 25 for females, and 24.5 for males, up from 22 and 21.7, respectively, in the $1970 \mathrm{~s} .{ }^{38}$ With global levels of smoking, and death rates from smoking decreasing over time, the negative health effects of overweight (BMI>25) and obesity (BMI>30) will continue to become increasingly relevant and significant during the course of this century. This has been acknowledged by governments and health organisations across the world, with many different strategies used to try to reduce the prevalence of obesity, with varying degrees of success. The increased risk of weight gain through reduced non-exercise activity thermogenesis is especially relevant given the increased morbidity and mortality among obese individuals affected by COVID-19..$^{39}$ It is therefore imperative to gain an understanding of current levels of sedentary behaviour among GPs, as well as to identify barriers and facilitators to GPs reducing their sedentary behaviour.

It has already been established that more physically active GPs are more likely to recommend physical activity to their patients and patients are more likely to make healthy lifestyle changes if they believe their GP follows the health advice themselves. ${ }^{17-21,22-24,28}$ Furthermore, given that GPs have such high levels of patient contact, reducing sedentary behaviour and increasing physical activity among GPs could lead to health benefits for both GPs themselves, at an individual level, and their patients, at a population level. This could play a vital role, as part of a multifaceted approach alongside public health initiatives and changes to the built environment, in creating a culture shift away from a society which is increasingly sedentary, to one which is increasingly physically active.

\section{Strengths}

By using an initial online questionnaire survey, this will allow the survey to be widely disseminated among GPs 
and GPSTs throughout Northern Ireland, which maximises the likelihood that all potential participants will be able to access the survey. This will allow both quantitative and qualitative data to be captured and stored securely for analysis and interpretation. Questionnaires have been used extensively to determine levels of sedentary behaviour among large population groups. Multi-item questionnaires with a relatively short recall period have been shown to be more reliable than single item questions and longer recall periods for the assessment of sedentary time. ${ }^{32}$

Using accelerometers with an accompanying sleep/work log among a smaller, purposive sample of participants, will allow objective, quantitative data to be gathered regarding levels of sedentary behaviour on work days and non-work days. Thigh-worn accelerometers are highly accurate for distinguishing between sedentary behaviour (such as sitting or lying down) and other behaviours (such as standing and physical activity). ${ }^{33}$

Carrying out semi-structured interviews among a smaller, purposive sample of participants from the accelerometer study will allow in-depth exploration of participants' thoughts, opinions and knowledge regarding sedentary behaviour and physical activity. Remote interviews by telephone or webcam are a convenient and cost-effective way of talking to people who are geographically dispersed. ${ }^{40}$

\section{Limitations}

Questionnaire surveys relying on self-reported estimation of sedentary time have been shown to have moderate to poor validity, and only moderate to good reliability compared to objective, accelerometer measured sedentary time. ${ }^{32}$ For this reason, questionnaire data will be correlated with objective, accelerometer measured sedentary behaviour.

Thigh-worn accelerometers are unable to detect upper body movement, so if a wearer is sitting, reclining or lying while performing exercise involving the trunk or arms, this may incorrectly be recorded as sedentary behaviour.

Semi-structured interviews will be conducted remotely by webcam or telephone, instead of in person. This may limit the rapport between researcher and participant, and inhibit interpretation of non-verbal communication and body language, which may lead to less qualitative detail. ${ }^{40}$

\section{CONCLUSION}

At present, there is a paucity of research examining current levels of sedentary behaviour among GPs. There is a need for objective data to determine their current levels of sedentary behaviour, in order to identify ways of reducing this, if possible. There is also currently a lack of qualitative research examining the potential barriers and facilitators to reducing sedentary behaviour among GPs. This is vital when considering interventions to reduce sedentary behaviour, to ensure any interventions would be practical and appropriate in the General Practice environment.

This paper has outlined a protocol for a sequential, mixedmethods study exploring sedentary behaviour among GPs and GPSTs in Northern Ireland. In the initial stages of this study, quantitative data will be obtained regarding current levels of sedentary behaviour among GPs and GPSTs. In the final stage of this study, qualitative data will be gathered regarding the knowledge, thoughts and opinions of GPs and GPSTs on the subject of sedentary behaviour. Findings of this study will shed further light on the new ways of working as a result of the COVID-19 pandemic, which will be relevant to clinicians working in similar primary care settings throughout the United Kingdom and internationally.

\section{ACKNOWLEDGEMENTS}

Authors would like to acknowledge the assistance of Professor Mark Tully and Dr. Jason Wilson, from Ulster University, for their input in the design of the accelerometer study.

Funding: This study is funded by the Health and Social Care Research and Development Division, Public Health Agency's GP Academic Research Training Scheme under grant number EAT/5332/19. The funding body had no influence in the design of the study nor in the collection, analysis and interpretation of data as well as the writing of related manuscripts

Conflict of interest: None declared

Ethical approval: An ethics application was submitted for the original study to the Faculty of Medicine, Health and Life Sciences Research Ethics Committee of Queen's University Belfast on 11 March 2020 (MHLS 20_39). This was approved on 07 April 2020. An amended ethics application for the modified study (due to changes required as a result of the COVID-19 pandemic) was submitted to the Faculty of Medicine, Health and Life Sciences Research Ethics Committee of Queen's University Belfast on 19 June 2020. This was approved on 03 July 2020 (MHLS 20_39 - Amendment 1). Written, informed consent to participate will be obtained by all participants prior to each stage of the study in accordance with General Data Protection Regulations (GDPR). This will be achieved using an online form

\section{REFERENCES}

1. Tremblay MS, Aubert S, Barnes JD, Saunders TJ, Carson V, Latimer-Cheung AE, et al. Sedentary Behavior Research Network (SBRN) - Terminology Consensus Project process and outcome. Int J Behav Nutr Physical Activity. 2017;14(1).

2. Hamer M, Stamatakis E, Steptoe A. Dose-response relationship between physical activity and mental health: the Scottish Health Survey. Br J Sports Med. 2009;43(14):1111-4. 
3. Rovio S, Kåreholt I, Helkala E-L, Viitanen M, Winblad B, Tuomilehto J, et al. Leisure-time physical activity at midlife and the risk of dementia and Alzheimer's disease. Lancet Neurol. 2005;4(11):705-11.

4. Biswas A, Oh PI, Faulkner GE, Bajaj RR, Silver MA, Mitchell MS, et al. Sedentary time and its association with risk for disease incidence, mortality, and hospitalization in adults: a systematic review and meta-analysis. Ann Intern Med. 2015;162(2):123-32.

5. Koster A, Caserotti P, Patel KV, Matthews CE, Berrigan D, Van Domelen DR, et al. Association of sedentary time with mortality independent of moderate to vigorous physical activity. PLoS One. 2012;7(6):e37696.

6. Levine JA. Interindividual Variation in Posture Allocation: Possible Role in Human Obesity. Science. 2005;307(5709):584-6.

7. Ekelund U, Steene-Johannessen J, Brown WJ, Fagerland MW, Owen N, Powell KE, et al. Does physical activity attenuate, or even eliminate, the detrimental association of sitting time with mortality? A harmonised meta-analysis of data from more than 1 million men and women. Lancet. 2016;388(10051):1302-10.

8. Ekelund U, Tarp J, Steene-Johannessen J, Hansen BH, Jefferis B, Fagerland MW, et al. Dose-response associations between accelerometry measured physical activity and sedentary time and all cause mortality: systematic review and harmonised metaanalysis. BMJ. 2019;366:14570.

9. Scarborough P, Bhatnagar P, Wickramasinghe KK, Allender S, Foster C, Rayner M. The economic burden of ill health due to diet, physical inactivity, smoking, alcohol and obesity in the UK: an update to 2006-07 NHS costs. J Public Health. 2011;33(4):527-35.

10. Ding D, Lawson KD, Kolbe-Alexander TL, Finkelstein EA, Katzmarzyk PT, Van Mechelen W, et al. The economic burden of physical inactivity: a global analysis of major non-communicable diseases. Lancet. 2016;388(10051):1311-24.

11. Heron L, O'Neill C, McAneney H, Kee F, Tully MA. Direct healthcare costs of sedentary behaviour in the UK. J Epidemiol Comm Health. 2019;73(7):625-9.

12. Davies SC, Atherton F, McBride M, Calderwood C. UK Chief Medical Officers' Physical Activity Guidelines 2019.

13. OECD. Physicians by category. 2020. Available at: http://www.oecd.org/health/health-data.htm. Accessed on: 20 October 2020.

14. NHS-England. NHS 5 Year Forward View - Primary Care. 2017. Available at: https://www .england.nhs.uk/five-year-forward-view/next-stepson-the-nhs-five-year-forward-view/primary-care/. Accessed on: 20 October 2020.

15. Croteau K, Schofield G, McLean G. Physical activity advice in the primary care setting: Results of a population study in New Zealand. Aus N Zeal J Public Health. 2006;30(3):262-7.
16. Short CE, Hayman M, Rebar AL, Gunn KM, De Cocker K, Duncan MJ, et al. Physical activity recommendations from general practitioners in Australia. Results from a national survey. Aus N Zeal J Public Health. 2016;40(1):83-90.

17. 17. Frank E, Segura C, Shen H, Oberg E. Predictors of Canadian Physicians' Prevention Counseling Practices. Canad J Public Health. 2010;101(5):390-5.

18. Klein D, Guenther C, Ross S. Do as I say, not as I do. Lifestyles and counseling practices of physician faculty at the University of Alberta. 2016;62(7):3939.

19. Lobelo F, de Quevedo IG. The evidence in support of physicians and health care providers as physical activity role models. Am J Lifestyle Med. 2016;10(1):36-52.

20. Lobelo F, Duperly J, Frank E. Physical activity habits of doctors and medical students influence their counselling practices. $\mathrm{Br} \mathrm{J}$ Sports Med. 2008;43(2):89-92.

21. Stanford FC, Durkin MW, Stallworth JR, Powell CK, Poston MB, Blair SN. Factors that Influence Physicians' and Medical Students' Confidence in Counseling Patients About Physical Activity. 2014;35(3):193-201.

22. Frank E, Breyan J, Elon L. Physician disclosure of healthy personal behaviors improves credibility and ability to motivate. Arch Family Med. 2000;9(3):287-9.

23. Fraser S, Leveritt M, Ball L. Patients' perceptions of their general practitioner's health and weight influences their perceptions of nutrition and exercise advice received. J Prim Health Care. 2013;5(4):3017.

24. Lemaire JB, Ewashina D, Polachek AJ, Dixit J, Yiu $\mathrm{V}$. Understanding how patients perceive physician wellness and its links to patient care: A qualitative study. PlOs one. 2018;13(5):e0196888.

25. Joy M, McGagh D, Jones N, Liyanage H, Sherlock J, Parimalanathan V, et al. Reorganisation of primary care for older adults during COVID-19: a crosssectional database study in the UK. Br J Gen Pract. 2020;70(697):540-7.

26. Keohane DM, McGillivary NA, Daly B. Physical activity levels and perceived barriers to exercise participation in Irish General Practitioners and General Practice trainees. Ir Med J. 2018;111(2):690.

27. Suija K, Pechter U, Maaroos J, Kalda R, Ratsep A, Oona M, et al. Physical activity of Estonian family doctors and their counselling for a healthy lifestyle: a cross-sectional study. BMC Fam Pract. 2010;11:48.

28. Brotons C, Björkelund C, Bulc M, Ciurana R, Godycki-Cwirko M, Jurgova E, et al. Prevention and health promotion in clinical practice: the views of general practitioners in Europe. 2005;40(5):595-601.

29. Pardo A, McKenna J, Mitjans A, Camps B, ArandaGarcía S, Garcia-Gil J, et al. Physical Activity Level and Lifestyle-Related Risk Factors From Catalan Physicians. 2014;11(5):922-9. 
30. Ivankova NV, Creswell JW, Stick SL. Using MixedMethods Sequential Explanatory Design: From Theory to Practice. Field Methods. 2006;18(1):3-20.

31. Prince SA, LeBlanc AG, Colley RC, Saunders TJ. Measurement of sedentary behaviour in population health surveys: a review and recommendations. Peer J. 2017;5:4130.

32. Prince SA, Cardilli L, Reed JL, Saunders TJ, Kite C, Douillette K, et al. A comparison of self-reported and device measured sedentary behaviour in adults: a systematic review and meta-analysis. Int $\mathbf{J}$ Behav Nutr Physical Activity. 2020;17(1):31.

33. Skotte J, Korshøj M, Kristiansen J, Hanisch C, Holtermann A. Detection of Physical Activity Types Using Triaxial Accelerometers. J Physical Activity Health. 2014;11(1):76-84.

34. Crowley P, Skotte J, Stamatakis E, Hamer M, Aadahl M, Stevens ML, et al. Comparison of physical behavior estimates from three different thigh-worn accelerometers brands: a proof-of-concept for the Prospective Physical Activity, Sitting, and Sleep consortium (ProPASS). Int J Behav Nutr Physical Activity. 2019;16(1):65.

35. Doherty A, Jackson D, Hammerla N, Plötz T, Olivier $\mathrm{P}$, Granat $\mathrm{MH}$, et al. Large Scale Population Assessment of Physical Activity Using Wrist Worn Accelerometers: The UK Biobank Study. PIOs one. 2017;12(2):e0169649.
36. Migueles JH, Cadenas-Sanchez C, Ekelund U, Delisle Nyström C, Mora-Gonzalez J, Löf M, et al. Accelerometer Data Collection and Processing Criteria to Assess Physical Activity and Other Outcomes: A Systematic Review and Practical Considerations. Sports Med. 2017;47(9):1821-45.

37. Sekhon M, Cartwright M, Francis JJ. Acceptability of healthcare interventions: an overview of reviews and development of a theoretical framework. BMC Health Services Res. 2017;17(1).

38. World Health Organization. BMI Classification. Global Database on Body Mass Index. 2014.

39. Simonnet A, Chetboun M, Poissy J, Raverdy V, Noulette J, Duhamel A, et al. High Prevalence of Obesity in Severe Acute Respiratory Syndrome Coronavirus-2 (SARS-CoV-2) Requiring Invasive Mechanical Ventilation. Obesity. 2020.

40. Bolderston A. Conducting a Research Interview. J Med Imaging Rad Sc. 2012;43(1):66-76.

Cite this article as: Mayne RS, Hart ND, Heron N. Exploration of sedentary behaviour among general practitioners: protocol for a mixed methods study. Int $\mathbf{J}$ Clin Trials 2021;8(1):51-7. 